\title{
Almost Alternating Sums
}

\author{
Kevin O’Bryant, Bruce Reznick, and Monika Serbinowska
}

1. INTRODUCTION. The behavior of the sum

$$
S_{N}(\alpha):=\sum_{n=1}^{N}(-1)^{\lfloor n \alpha\rfloor}
$$

as $N \rightarrow \infty$ is not transparent (here $\lfloor x\rfloor$ signifies the greatest integer not larger than $x)$. The random walk $\sum_{n=1}^{N} w_{n}$, where the $w_{n}$ are independent random variables taking the values 1 and -1 with equal probability, is known 24 to typically have absolute value around $c \sqrt{N}$ for an appropriate constant $c$ and large $N$. Knowing this and knowing also that for irrational $\alpha$ the sequence $\lfloor n \alpha\rfloor$ is "random-ish" modulo 2 , a natural guess is that $\left|S_{N}(\alpha)\right|$ is also around $\sqrt{N}$.

Contrary to this expectation, for almost all real numbers $\alpha$

$$
\left|S_{N}(\alpha)\right| \leq(\log N)^{2}
$$

for all large $N$. This is a corollary of a theorem of Khintchine, which we state precisely in section 2 .

We devote the bulk of this article to two elementary proofs that

$$
\left|S_{N}(\alpha)\right| \leq \frac{\log N}{2 \log (1+\sqrt{2})}+1
$$

for all $N$ and an explicit countable set of $\alpha$, including $\sqrt{2}$ and $\sqrt{5}+1$. Our first proof is entirely self-contained and is given in section 3 . In section 4 , we give a second proof that, while elementary, makes use of continued fractions. This proof applies to an uncountable set of $\alpha$ including, for example, $2 /(e-1)$. Moreover, it can be adapted to show that there are infinitely many $N$ such that

$$
\left|S_{N}(\sqrt{2})\right|>\frac{\log N}{2 \log (1+\sqrt{2})}+0.78 \text {. }
$$

This means that as $N \rightarrow \infty$ the first constant in (2) is sharp (at least for $\alpha=\sqrt{2}$ ) and the second cannot be improved even to $3 / 4$.

The situation for rational $\alpha$ is more clear. As shown by the third author [23], for rational $\alpha$ the limit

$$
\lim _{N \rightarrow \infty} \frac{S_{N}(\alpha)}{N}
$$

is well-defined. Moreover, if this limit is zero, then $S_{N}(\alpha)$ is bounded and periodic. 


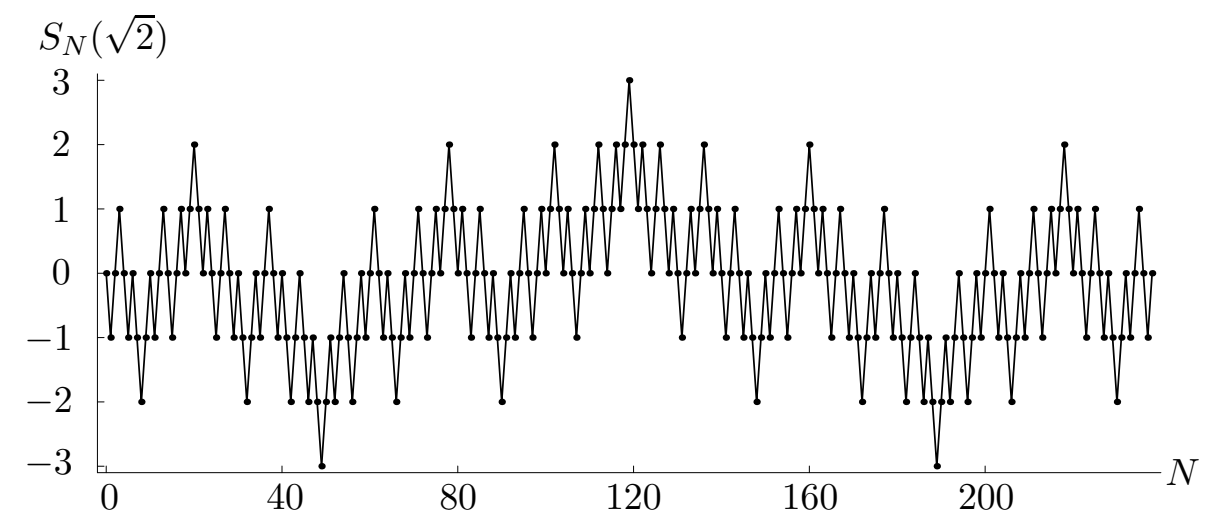

Figure 1: The points $\left(N, S_{N}(\sqrt{2})\right)$ have been connected for visual clarity.

In Figure 11, we show the points $\left(N, S_{N}(\sqrt{2})\right)$ when $0 \leq N \leq 238$. It is already apparent in this figure that $S_{N}(\sqrt{2})$ is not behaving like a random walk: there are never three consecutive "up" steps. Also, the graph is symmetric around the peak at $N=119$ (i.e., $S_{N}(\sqrt{2})=S_{238-N}(\sqrt{2})$ for those $N$ pictured in Figure 1).

If we restrict our attention to just the record-holders - those $N$ for which $S_{N}(\sqrt{2})$ takes on a value for the first time -another aspect of the structure of $S_{N}(\sqrt{2})$ becomes apparent. For the sake of rigor, we define the record-holder at the integer $k$ by

$$
R_{k}(\alpha)=\min \left\{N \geq 0: S_{N}(\alpha)=k\right\} .
$$

In Figure 2, we plot the points $\left(\log R_{k}(\sqrt{2}), k\right.$ ) when $-9 \leq k \leq 9$ (except $k=$ $0)$. The points approach two lines; we show in Corollary 4.5 that $R_{k}(\sqrt{2})$ is $\left\lfloor\frac{1}{4}(\sqrt{2}+1)^{2 k+1}\right\rfloor$ if $k$ is positive and $\left\lfloor\frac{1}{4}(\sqrt{2}+1)^{-2 k}\right\rfloor$ if $k$ is negative.

Our proofs do not give a logarithmic bound on $\left|S_{N}(\alpha)\right|$ for general $\alpha$; indeed, for $\alpha=\pi \approx 3.14159$ we do not believe that a logarithmic bound is correct. When $N \leq 10^{7}$, computations reveal that $-22 \leq S_{N}(\pi) \leq 3$. We are unaware of any nontrivial bound on $\left|S_{N}(\pi)\right|$. The record holders $R_{k}(\pi)$ are plotted in Figure 3 , We note that the asymmetry and irregular clumping of points in Figure 3 seems to be more typical than the orderliness depicted in Figure 2

In section 2, we prove that $\left|S_{N}(\alpha)\right| \leq C_{\alpha} \log N$ (for quadratic irrational $\alpha$ ) using the theory of discrepancy, which we define but do not pursue further. Section 2 is provided for historical background and as a hook into related literature; the remainder of this article is logically independent of section 2 . In section 3 , we give an elementary bound on $\left|S_{N}(\alpha)\right|$ for a countable set of $\alpha$. In section 4 , we introduce the required facts and definitions about continued fractions and give a formula for $S_{N}(\alpha)$ for an uncountable set of $\alpha$. From this formula, we get upper and lower bounds on the growth of $S_{N}(\alpha)$ and prove the formulas for $R_{k}(\sqrt{2})$ stated earlier. We conclude in section 5 with some questions that we have not been able to answer.

Before we begin the analysis, we introduce some notation. The natural numbers begin at 1 (i.e., $\mathbb{N}=\{1,2,3, \ldots\}$ ). The fractional part of $x$ is given by $\{x\}:=x-\lfloor x\rfloor$. 


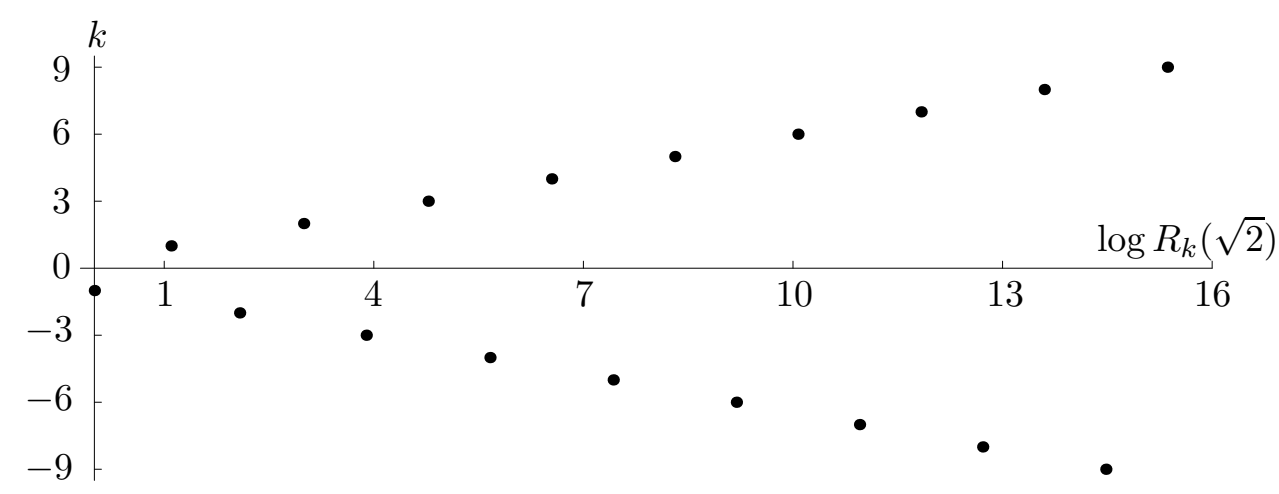

Figure 2: The points $\left(\log R_{k}(\sqrt{2}), k\right)$ when $-9 \leq k \leq 9$, except $k=0$.

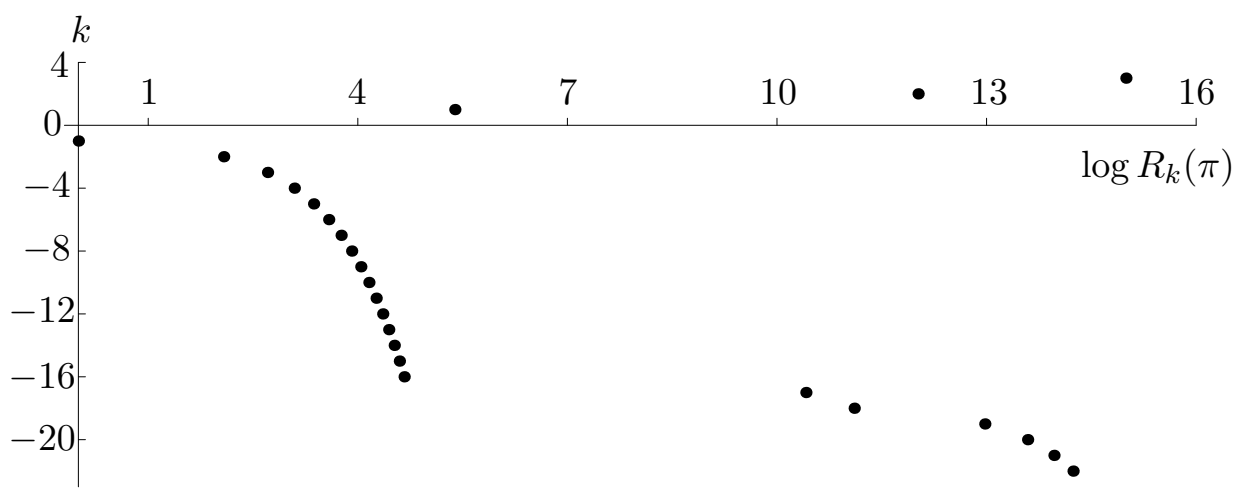

Figure 3: The points $\left(\log R_{k}(\pi), k\right)$, when $-22 \leq k \leq 3$, except $k=0$.

We make use of big-O notation only with respect to $N$; that is, $f(N, \alpha)=\mathcal{O}(g(N))$ if there is a constant $C$ such that $|f(N, \alpha)| \leq C g(N)$ for all sufficiently large $N$. Note that $C$ may depend on $\alpha$ but does not depend on $N$. We also make lavish use of Iverson's notation

$$
\llbracket Q \rrbracket:= \begin{cases}1 & \text { if the statement } Q \text { is true; } \\ 0 & \text { if the statement } Q \text { is false. }\end{cases}
$$

See Knuth [15] for an eloquent, award-winning argument in favor of this notation. ${ }^{1}$ Iverson used $(Q)$, and Knuth uses $[Q]$, but we find $\llbracket Q \rrbracket$ visually appealing, especially in light of the other frequent uses for parentheses and brackets.

2. DISCREPANCY. An enduring topic in number theory has been the discrepancy shown between the expected and actual behavior of a sequence in the interval $[0,1)$. For example, if a sequence $\left(w_{n}\right)$ is uniformly random, one would expect that

\footnotetext{
${ }^{1}$ See http://www.maa.org/awards/ford.html for a description of the Lester R. Ford Award for mathematical exposition and the list of award-winning articles.
} 
it is in the interval $[0, x)$ with frequency $x$. The difference between the actual and expected behavior is formally measured by

$$
D_{N}^{*}\left(a_{n}, x\right):=\left|x-\frac{1}{N} \sum_{n=1}^{N} \llbracket w_{n}<x \rrbracket\right|,
$$

and the discrepancy of the sequence is denoted

$$
D_{N}^{*}\left(a_{n}\right):=\sup _{0 \leq x<1} D_{N}^{*}\left(w_{n}, x\right)
$$

Discrepancy plays no role in the proofs of (21) given in sections 3 and 4 . However, the powerful results (stated below) of Behnke, Ostrowski and Hardy \& Littlewood, and Khintchine regarding the discrepancy of $(\{n \alpha\})$ imply bounds on $S_{N}(\alpha)$ that are more general but less precise than those given elsewhere in this article.

We now show the connection between $S_{N}(\alpha)$ and the discrepancy of fractional part sequences. First, observe that $\lfloor n \alpha\rfloor$ is even exactly when there is an integer $k$ with $2 k \leq n \alpha<2 k+1$, which is the same as $k \leq n \alpha / 2<k+1 / 2$. In other words,

$$
\llbracket\lfloor n \alpha\rfloor \text { is even } \rrbracket=\llbracket k \leq n \alpha / 2<k+1 / 2 \rrbracket=\llbracket\{n \alpha / 2\}<1 / 2 \rrbracket
$$

With one eye on the definition of $D_{N}^{*}(\alpha)$, we now rewrite the definition of $S_{N}(\alpha)$ using $(-1)^{\lfloor n \alpha\rfloor}=\llbracket\lfloor n \alpha\rfloor$ is even $\rrbracket-\llbracket\lfloor n \alpha\rfloor$ is odd $\rrbracket$ :

$$
S_{N}(\alpha)=\sum_{n=1}^{N} \llbracket\lfloor n \alpha\rfloor \text { is even } \rrbracket-\llbracket\lfloor n \alpha\rfloor \text { is odd } \rrbracket .
$$

We add this equation to the obvious

$$
N=\sum_{n=1}^{N} \llbracket\lfloor n \alpha\rfloor \text { is even } \rrbracket+\llbracket\lfloor n \alpha\rfloor \text { is odd } \rrbracket
$$

to arrive at

$$
S_{N}(\alpha)=2\left(\sum_{n=1}^{N} \llbracket\lfloor n \alpha\rfloor \text { is even } \rrbracket\right)-N=-2 N\left(\frac{1}{2}-\frac{1}{N} \sum_{n=1}^{N} \llbracket\{n \alpha / 2\}<1 / 2 \rrbracket\right) .
$$

Thus,

$$
\left|S_{N}(\alpha)\right|=2 N D_{N}^{*}(\{n \alpha / 2\}, 1 / 2) \leq 2 N D_{N}^{*}(\{n \alpha / 2\}) .
$$

There are several accounts of the theory underlying discrepancy, most notably the colorful introductory book of Hlawka [12] and the recent - and encyclopedictreatise of Drmota and Tichy [7. There are three results that are of particular interest here.

- Behnke [2], 3], 7] Corollary 1.65], 22] classified those $\alpha$ for which

$$
D_{N}^{*}(\{n \alpha\})=\mathcal{O}\left(\frac{\log N}{N}\right) .
$$

In particular, this is true of all quadratic irrationals and is not true of $1 /(e-1)$. 
- Ostrowski and Hardy \& Littlewood [7, Theorem 1.51] showed that for any $\alpha$ there are infinitely many $N$ with

$$
D_{N}^{*}(\{n \alpha\})>\frac{1}{100} \frac{\log N}{N} .
$$

- Let $\psi$ be any positive increasing function on $\mathbb{N}$. Khintchine [7, Theorem 1.72], [13, 14] proved that

$$
D_{N}^{*}(\{n \alpha\})=\mathcal{O}\left(\frac{\log N}{N} \psi(\log N)\right)
$$

for almost all real numbers $\alpha$ if and only if $\sum_{n=1}^{\infty}(n \psi(n))^{-1}$ converges.

The relations (3) imply that $S_{N}(\alpha)=\mathcal{O}(\log N)$ if $D_{N}^{*}(\{n \alpha / 2\})=\mathcal{O}(\log N / N)$, but not necessarily vice versa. Since $\sqrt{2}$ and $\sqrt{5}+1$ are quadratic irrationals, Behnke's result implies that $S_{N}(\sqrt{2})$ and $S_{N}(\sqrt{5}+1)$ have logarithmic bounds, but not that $S_{N}(2 /(e-1))$ does. Schoißengeier's work [22] can be used to find

$$
\limsup _{N \geq 1} \frac{D_{N}^{*}(\{n \alpha / 2\})}{\log N}
$$

for any specific quadratic irrational, but this is typically strictly larger than

$$
\limsup _{N \geq 1} \frac{\left|S_{N}(\alpha)\right|}{\log N}
$$

To be fair, we note that the discrepancy approach says that $S_{N}(\sqrt{3})$ has a logarithmic bound; this does not follow from our work in the next sections.

In light of Schimdt's result, it is impossible to use a discrepancy bound to prove a bound on $S_{N}(\alpha)$ that is sublogarithmic. As a result of Corollary 4.5, however, for any function $\psi(N)$ with $\psi(1) \geq 1$ that increases to infinity we can find an $\alpha$ with $\left|S_{N}(\alpha)\right| \leq \psi(N)$ for all $N$. For example,

$$
S_{N}\left(\frac{2}{e-1}\right)=\mathcal{O}\left(\frac{\log N}{\log \log N}\right) .
$$

Khintchine's result and (3) imply that for almost all real numbers $\alpha$

$$
S_{N}(\alpha)=\mathcal{O}(\log N \psi(\log N))
$$

if $\sum_{n=1}^{\infty}(n \psi(n))^{-1}<\infty$. In particular, set $\psi(n)=\sqrt{n}$ and observe that if $f(N)=$ $\mathcal{O}\left((\log N)^{3 / 2}\right)$, then for all large $N$ we have $|f(N)| \leq(\log N)^{2}$. This verifies the rough statement of (11). It is conceivable that $S_{N}(\alpha)=\mathcal{O}(\log N)$ for almost all $\alpha$, but we find this unlikely.

3. TWO IDEAS. The sequence $S_{N}(\alpha)$ has several near-symmetries, which we formally state and prove as "ideas." In this section, we explicitly state and prove two of them, and show how they can be combined to prove Theorem [3.1. Inequality (2) is an immediate consequence of Theorem [3.1. We note that Theorem 3.1] is substantively identical to the main theorem in [4, although both our statement and proof are simpler. 
Theorem 3.1. If $m$ is a positive integer, then

$$
\left|S_{N}\left(\sqrt{m^{2}+1}-m+1\right)\right| \leq \frac{\log N}{2 \log \left(\sqrt{m^{2}+1}+m\right)}+1 .
$$

Our first idea is a combination of Beatty's theorem and a peculiar renormalization of $S_{N}(\alpha)$. We first state Beatty's theorem, and for the sake of making this section entirely self-contained we give the "book" proof of Ostrowski and Hyslop [1] first published in this Monthly in 1927. Then we give the renormalization, and then our two ideas.

Lemma 3.2 (Beatty's theorem). If $\alpha>1$ is irrational and $1 / \alpha+1 / \beta=1$, then the sequences $(\lfloor n \alpha\rfloor)$ and $(\lfloor n \beta\rfloor)$ partition $\mathbb{N}$.

Proof. Let $N$ be a natural number. We will show that there is exactly one term of the two sequences $(n \alpha)$ and $(n \beta)$ between $N$ and $N+1$. Since $N \alpha$ is not an integer, there are $\lfloor N / \alpha\rfloor$ multiples of $\alpha$ less than $N$, and likewise there are $\lfloor N / \beta\rfloor$ multiples of $\beta$. Therefore, from the two sequences there are exactly $\lfloor N / \alpha\rfloor+\lfloor N / \beta\rfloor$ terms strictly less than $N$. After writing

$N-2=N\left(\frac{1}{\alpha}+\frac{1}{\beta}\right)-2=\left(\frac{N}{\alpha}-1\right)+\left(\frac{N}{\beta}-1\right)<\left\lfloor\frac{N}{\alpha}\right\rfloor+\left\lfloor\frac{N}{\beta}\right\rfloor<\frac{N}{\alpha}+\frac{N}{\beta}=N$,

we see that $N-2<\lfloor N / \alpha\rfloor+\lfloor N / \beta\rfloor<N$. In other words, there are exactly $\lfloor N / \alpha\rfloor+\lfloor N / \beta\rfloor=N-1$ terms below $N$. Applying the same reasoning with $N+1$ in place of $N$, we find that there are exactly $N$ terms below $N+1$. Thus, as claimed, there is exactly one term between $N$ and $N+1$.

And now for our peculiar renormalization. Let

$$
S(\alpha ; x):=\frac{1}{4}+\sum_{0<n \alpha \leq x}(-1)^{\lfloor n \alpha\rfloor}=\frac{1}{4}+\sum_{n=1}^{\lfloor x / \alpha\rfloor}(-1)^{\lfloor n \alpha\rfloor} .
$$

In terms of our earlier notation, $S_{N}(\alpha)=S(\alpha ; N \alpha)-1 / 4$. We have achieved two things with this definition. First, we have introduced " $1 / 4$ " at a strategic moment (with 20-20 hindsight). Second, the parameter $x$ is naturally scaled for multiples of $\alpha$. This is important, since the sums $S(\alpha ; x)$ and $S(\beta ; x)$ (with $1 / \alpha+1 / \beta=1$ ) are almost complementary. We invite the dubious reader to rewrite Idea 1 in terms of $S_{N}(\alpha)$.

Idea 1. If $\alpha>1$ is irrational and $1 / \alpha+1 / \beta=1$, then $S(\alpha ; x)+S(\beta ; x)= \pm 1 / 2$.

Proof. Since $\alpha$ and $\beta$ satisfy the hypotheses of Beatty's theorem, the set of all integers $\lfloor n \alpha\rfloor,\lfloor n \beta\rfloor$-where $n \alpha$ and $n \beta$ are in $(0, x]$-is the set $\{1,2, \ldots, r\}$, where $r$ may be either $\lfloor x\rfloor$ or $\lfloor x\rfloor-1$. Thus, we write the deepest equation in this section:

$$
S(\alpha ; x)+S(\beta ; x)=\frac{2}{4}+\sum_{j=1}^{r}(-1)^{j} .
$$

The right-hand side is $\pm 1 / 2$, depending on the parity of $r$. 
Our second idea is really little more than the inauspicious observation that $(-1)^{2}=1$.

Idea 2. If $m$ is a natural number and $\beta>2 m$, then $S(\beta ; x)=S\left(\beta-2 m ; \frac{\beta-2 m}{\beta} x\right)$.

Proof. By hypothesis $\beta>2 m>0$, so we can multiply inequalities by $(\beta-2 m) / \beta$ :

$$
\llbracket 0<n \beta \leq x \rrbracket=\llbracket 0<n(\beta-2 m) \leq \frac{\beta-2 m}{\beta} x \rrbracket .
$$

Since $\lfloor n(\beta-2 m)\rfloor=\lfloor n \beta\rfloor-2 n m \equiv\lfloor n \beta\rfloor(\bmod 2)$, we know that

$$
\begin{aligned}
S(\beta ; x) & =\frac{1}{4}+\sum_{0<n \beta \leq x}(-1)^{\lfloor n \beta\rfloor} \\
& =\frac{1}{4}+\sum_{0<n(\beta-2 m) \leq \frac{\beta-2 m}{\beta} x}(-1)^{\lfloor n(\beta-2 m)\rfloor} \\
& =S\left(\beta-2 m ; \frac{\beta-2 m}{\beta} x\right) .
\end{aligned}
$$

Proof of Theorem [3.1. For the sake of being specific we work with $m=1$, setting $\alpha=\sqrt{2}$. Afterwards, we indicate the minor changes needed for general $m$. Since we wish to apply Idea 11 and $\frac{1}{\sqrt{2}}+\frac{1}{\sqrt{2}+2}=1$, we set $\beta=\sqrt{2}+2$. In this notation, Idea 1 becomes

$$
|S(\alpha ; x)| \leq|S(\beta ; x)|+\frac{1}{2},
$$

and Idea 2 (with $m=1$ ) becomes

$$
S(\beta ; x)=S(\alpha ;(\sqrt{2}-1) x) .
$$

Set $\lambda=\sqrt{2}-1$. Combining these two ideas and iterating, we find that

$$
\begin{aligned}
|S(\alpha ; x)| \leq|S(\beta ; x)|+\frac{1}{2} & =|S(\alpha ; \lambda x)|+\frac{1}{2} \\
& \leq\left|S\left(\alpha ; \lambda^{2} x\right)\right|+\frac{1}{2}+\frac{1}{2} \\
& \vdots \\
& \leq\left|S\left(\alpha ; \lambda^{k} x\right)\right|+\frac{k}{2} .
\end{aligned}
$$

If $k>\log _{\lambda}(\alpha / x)$, then $\lambda^{k} x<\alpha$, so $S\left(\alpha ; \lambda^{k} x\right)=1 / 4$. Setting $k=1+\left\lfloor\log _{\lambda}(\alpha / x)\right\rfloor$ leads us to the inequality

$$
|S(\alpha ; x)| \leq \frac{1}{4}+\frac{k}{2}=\frac{1}{4}+\frac{1}{2}\left(1+\left\lfloor\frac{\log (\alpha / x)}{\log \lambda}\right\rfloor\right) \leq \frac{\log (x / \alpha)}{2 \log \left(\lambda^{-1}\right)}+\frac{3}{4} .
$$

Since $\left|S_{N}(\alpha)\right|=|S(\alpha ; N \alpha)-1 / 4| \leq|S(\alpha ; N \alpha)|+1 / 4$,

$$
\left|S_{N}(\alpha)\right| \leq \frac{\log N}{2 \log \left(\lambda^{-1}\right)}+1
$$


as claimed. For general $m$, set $\alpha_{m}=\sqrt{m^{2}+1}+m-1, \beta_{m}=\sqrt{m^{2}+1}+m+1$, and $\lambda_{m}=\sqrt{m^{2}+1}-m$. The proof goes through verbatim.

4. THIRD IDEA. Our third idea starts from the observation that if $\alpha$ is rational, say $\alpha=p / q$, then $(-1)^{\lfloor n \alpha\rfloor}$ is periodic. In particular, if $N>q$ then

$$
\begin{aligned}
S_{N}(p / q) & =\sum_{n=1}^{q}(-1)^{\lfloor n p / q\rfloor}+\sum_{n=1}^{N-q}(-1)^{\lfloor(q+n) p / q\rfloor} \\
& =\sum_{n=1}^{q}(-1)^{\lfloor n p / q\rfloor}+\sum_{n=1}^{N-q}(-1)^{p+\lfloor n p / q\rfloor}=S_{q}(p / q)+(-1)^{p} S_{N-q}(p / q) .
\end{aligned}
$$

This allows us to replace $S_{N}$ with the shorter sums $S_{q}$ and $S_{N-q}$, and if $N-q>q$, we can replace $S_{N-q}$ with an even shorter sum.

If $\alpha$ is irrational, then $(-1)^{\lfloor n \alpha\rfloor}$ is not periodic, but if $\alpha$ is "close" to $p / q$, then $(-1)^{\lfloor n \alpha\rfloor}$ should be "close" to periodic. This is our third idea. Before we state it quantitatively, though, we need to introduce simple continued fractions and the continued fraction expansion. Most books on elementary number theory have chapters on continued fractions. The base- $\alpha$ continued fraction expansion of an integer (which we define below) is dealt with in [8] and [22, among other places.

Continued fractions. Throughout this article, $a_{i}$ always signifies an integer; if $i>0$, then $a_{i}$ is positive. We define the function $[\cdot]$ by $\left[a_{0}\right]=a_{0}$ and for $r \geq 1$

$$
\left[a_{0} ; a_{1}, a_{2}, \ldots, a_{r}\right]=a_{0}+\frac{1}{\left[a_{1} ; a_{2}, \ldots, a_{r}\right]}=a_{0}+\frac{1}{a_{1}+\frac{1}{a_{2}+\ddots+\frac{1}{a_{r}}}} .
$$

For every rational $p / q$ there is a unique $r$ and a unique sequence $a_{0}, a_{1}, \ldots, a_{r}$ with $a_{r} \geq 2$ and $p / q=\left[a_{0} ; a_{1}, \ldots, a_{r}\right]$.

The limit

$$
\left[a_{0} ; a_{1}, a_{2}, \ldots\right]:=\lim _{r \rightarrow \infty}\left[a_{0} ; a_{1}, a_{2}, \ldots, a_{r}\right]
$$

always exists and is always irrational. Moreover, for each irrational $\alpha$ there is a unique sequence $a_{0}, a_{1}, \ldots$ with $\alpha=\left[a_{0} ; a_{1}, \ldots\right]$; we call $\left[a_{0} ; a_{1}, \ldots\right]$ the continued fraction of $\alpha$, and the $a_{t}(t \geq 0)$ the partial quotients of $\alpha$.

Given $\alpha$, one can compute the $a_{i}$ by noting that $a_{0}=\lfloor\alpha\rfloor$ and inductively using the equation $\alpha=a_{0}+\left[a_{1} ; a_{2}, \ldots\right]^{-1}$. For example, $\left[a_{1} ; a_{2}, \ldots\right]=\left(\alpha-a_{0}\right)^{-1}$, so $a_{1}=\left\lfloor\left(\alpha-a_{0}\right)^{-1}\right\rfloor$, and now $\left[a_{2} ; a_{3}, \ldots\right]=\left(\left(\alpha-a_{0}\right)^{-1}-a_{1}\right)^{-1}$. The easiest concrete example is $\alpha=\sqrt{2}+1$. We have $a_{0}=\lfloor\sqrt{2}+1\rfloor=2$, and $\sqrt{2}+1=2+\left[a_{1} ; a_{2}, \ldots\right]^{-1}$, whence $\left[a_{1} ; a_{2}, \ldots\right]=(\sqrt{2}-1)^{-1}=\sqrt{2}+1$ and $a_{1}=\lfloor\sqrt{2}+1\rfloor=2$. Iterating, we find that $\sqrt{2}+1=[2 ; 2,2,2, \ldots]$. We note the minor variation

$$
\sqrt{2}=[1 ; 2,2,2, \ldots]
$$


and the generalization

$$
\alpha_{m}=\sqrt{m^{2}+1}-m+1=[1 ; 2 m, 2 m, 2 m, \ldots] \quad(m \in \mathbb{N}),
$$

which we leave as an exercise for the reader. A more difficult example is

$$
\frac{2}{e-1}=[1 ; 6,10,14,18, \ldots]
$$

which the very industrious can derive from the Taylor expansion of $e^{x}$ at $x=1$ :

$$
e=\sum_{n=0}^{\infty} \frac{1}{n !}=1+\frac{1}{2}\left(1+\frac{1}{3}\left(1+\frac{1}{4}(1+\cdots)\right)\right) .
$$

A difficult theorem of Behnke [7, Corollary 1.65] states that $D_{N}^{*}(\alpha)=\mathcal{O}(\log N / N)$ if and only if the sequence $\left(n^{-1} \sum_{i=1}^{n} a_{i}\right)$ is bounded, where $\alpha=\left[a_{0} ; a_{1}, a_{2}, \ldots\right]$. This is true of $\alpha_{m} / 2$, and indeed of every quadratic irrational, but is not true of $1 /(e-1)$. In fact, the set of $\alpha$ to which this applies has measure zero, but there are few well-known irrationals for which it is known whether the sequence $\left(n^{-1} \sum_{i=1}^{n} a_{i}\right)$ is bounded. For example, two famous problems are to determine the status of $\pi$ (see [11]) and $2^{1 / 3}$ (see [9, Problem F22]). We showed in section 2 that $\left|S_{N}(\alpha)\right| \leq 2 N D_{N}^{*}(\{\alpha / 2\})$, so this tells us that $S_{N}(\alpha)=\mathcal{O}(\log N)$ if the partial quotients of $\alpha / 2$ are bounded in average. There are $\alpha$, however, such that $S_{N}(\alpha)$ has a logarithmic bound yet $2 N D_{N}^{*}(\{n \alpha / 2\})$ does not.

We now inductively define two sequences using the partial quotients of $\alpha$.

$$
\begin{aligned}
& p_{-2}=0, \quad p_{-1}=1, \quad p_{i}=a_{i} p_{i-1}+p_{i-2}, \\
& q_{-2}=1, \quad q_{-1}=0, \quad q_{i}=a_{i} q_{i-1}+q_{i-2},
\end{aligned}
$$

The $q_{i}$ are called the continuants of $\alpha$. The remarkable (albeit elementary) fact here is that

$$
\frac{p_{i}}{q_{i}}=\left[a_{0} ; a_{1}, a_{2}, \ldots, a_{i}\right] .
$$

The rationals $p_{i} / q_{i}$ are called the convergents to $\alpha$. The general utility of continued fractions lies not in the convergence of the convergents to $\alpha$, but in the fact that $p_{i} / q_{i}$ is the closest rational to $\alpha$ with denominator less than $q_{i+1}$. This is so important that we state a strong form of this principle explicitly as Lemma 4.3. For a proof, we refer the reader to [10, proof of Theorem 182], [16, Theorem 7.13], or [18, Theorem $10.15]$.

Lemma 4.3. If $0<n<q_{i+1}$, then $\left|q_{i} \alpha-p_{i}\right| \leq\{n \alpha\} \leq 1-\left|q_{i} \alpha-p_{i}\right|$, with equality only if $n=q_{i}$.

There are several equivalent definitions of the base- $\alpha$ continued fraction expansion (CFE) of a nonnegative integer $N$. Fix $\alpha$, and let $q_{i}$ denote the continuants of $\alpha$. We define the CFE of $N$ to be the lexicographically first sequence $\left(Z_{i}\right)$ of nonnegative integers satisfying

$$
N=\sum_{i=0}^{\infty} Z_{i} q_{i}
$$


In other words, write $N$ as a sum of continuants greedily (i.e., always using the largest possible), and set $Z_{i}$ to be the number of times you used $q_{i}$. The following definitions are equivalent and useful:

- The CFE of 0 is $0,0,0, \ldots$, and the CFE of $N-q_{I}$ is

$$
Z_{0}, \ldots, Z_{I-1}, Z_{I}-1, Z_{I+1}, \ldots
$$

where $q_{I}$ is the largest continuant less than or equal to $N$.

- The $Z_{i}$ are nonnegative integers satisfying (4) and $\sum_{i=0}^{j} Z_{i} q_{i}<q_{j+1}$ whenever $j \geq 0$.

- The $Z_{i}$ are integers satisfying (4) and $0 \leq Z_{i} \leq a_{i+1}-\llbracket Z_{i-1}>0 \rrbracket$ whenever $i \geq 0$.

The continuants $q_{0}, q_{1}, q_{2}, \ldots$ are positive and increasing. Therefore, if $I$ is such that $q_{I}>N$, then $Z_{i}=0$ once $i \geq I$. We denote the CFE of $N$ by

$$
N=\left(Z_{I}, Z_{I-1}, \ldots, Z_{1}, Z_{0}\right) .
$$

Note that we have reversed the order of the $Z_{i}$; this is analogous to the custom of writing $\mathbf{4}+\mathbf{3} \cdot 10+\mathbf{2} \cdot 10^{2}$ as 234 .

We now compute the CFEs of 100 and of $10^{11}$ with $\alpha=2 /(e-1)=[1 ; 6,10,14, \ldots]$. Minding our $p$ s and $q \mathrm{~s}$, we make the following table.

\begin{tabular}{|c|ccccccccc|}
\hline$i$ & 0 & 1 & 2 & 3 & 4 & 5 & 6 & 7 & 8 \\
\hline$p_{i}$ & 1 & 7 & 71 & 1001 & 18089 & 398959 & 10391023 & 312129649 & 10622799089 \\
\hline$q_{i}$ & 1 & 6 & 61 & 860 & 15541 & 342762 & 8927353 & 268163352 & 9126481321 \\
\hline
\end{tabular}

The largest continuant not larger than 100 is 61 ; continuing greedily we find that $100=\mathbf{1} \cdot 61+\mathbf{6} \cdot 6+\mathbf{3} \cdot 1$. Thus, $100=(1,6,3)$. We also have $100=\mathbf{1 6} \cdot 6+$ $4 \cdot 1$, but $100 \neq(16,4)$ since this is not a valid CFE (the sequence $16,4,0,0, \ldots$ is lexicographically after $1,6,3,0, \ldots)$. A bit more arithmetic reveals that

$$
10^{11}=(10,32,17,6,8,15,11,9,0),
$$

a fact that we make use of in another example below.

Idea 3. If $p / q$ is the convergent to $\alpha$ with $q(<N)$ maximal, then

$$
S_{N}(\alpha)=S_{q}(\alpha)+(-1)^{p} S_{N-q}(\alpha) .
$$

Proof. Define $I$ by $q=q_{I}$. We show that $\lfloor\{n \alpha\}+q \alpha-p\rfloor=0$ when $0<n<q_{I+1}$ by demonstrating that

$$
0<\{n \alpha\}+q \alpha-p<1
$$

for $n$ in this range. If $n \neq q$, then from Lemma 4.3 we learn that

$$
-(q \alpha-p) \leq|q \alpha-p|<\{n \alpha\}<1-|q \alpha-p| \leq 1-(q \alpha-p),
$$


which verifies the inequalities (51). If $n=q$, then either $q \alpha-p=\{n \alpha\}$ or $q \alpha-p=$ $\{n \alpha\}-1$. In the first case, $0<q \alpha-p<1 / 2$ and $\{n \alpha\}+q \alpha-p=2(q \alpha-p)$ lies in $(0,1)$; in the second case, $-1 / 2<q \alpha-p<0$ and $\{n \alpha\}+q \alpha-p=2(q \alpha-p)+1$ belongs to $(0,1)$. Either way, we have verified the inequalities (5).

Since $N-q<N \leq q_{I+1}$, we have

$$
\begin{aligned}
& S_{N}(\alpha)-S_{q}(\alpha)=\sum_{n=1}^{N-q}(-1)^{\lfloor(n+q) \alpha\rfloor}=\sum_{n=1}^{N-q}(-1)^{\lfloor\lfloor n \alpha\rfloor+\{n \alpha\}+q \alpha-p+p\rfloor} \\
& =\sum_{n=1}^{N-q}(-1)^{\lfloor n \alpha\rfloor}(-1)^{\lfloor\{n \alpha\}+q \alpha-p\rfloor}(-1)^{p}=(-1)^{p} \sum_{k=1}^{N-q}(-1)^{\lfloor n \alpha\rfloor}=(-1)^{p} S_{N-q}(\alpha) .
\end{aligned}
$$

A formula for $S_{N}(\alpha)$. We are now in position to establish the following theorem. First we state it, then we give an example of it, and then we prove it. In the next subsection, we use Theorem 4.4 to describe the record-holders $R_{k}(\alpha)$ for those $\alpha$ satisfying the hypothesis of Theorem 4.4 .

Theorem 4.4. Let $\alpha=\left[a_{0} ; a_{1}, a_{2}, \ldots\right]$, with $a_{0}$ odd and all other $a_{i}$ even. If a natural number $N$ has base- $\alpha$ CFE $\left(Z_{I}, Z_{I-1} \ldots, Z_{1}, Z_{0}\right)$, then

$$
S_{N}(\alpha)=-\sum_{i=0}^{I}\left(\prod_{t=i+1}^{I}(-1)^{Z_{t}}\right) \llbracket i \text { even } \rrbracket \llbracket Z_{i} \text { odd } \rrbracket .
$$

We note that this formula can be quickly pulled out of Brown's decomposition (which we won't state) of Sturmian words (which we won't define); indeed, this is how the authors first found Theorem 4.4 A similar result, but applicable only to $\sqrt{2}$, appears in [6, section 5]. The proof there uses ideas similar to those used by Brown [5]. Our proof of Theorem 4.4 borrows from the proof of Brown's decomposition given by the first author in [17].

An example will illustrate the power this result harnesses. We compute $S_{N}(\alpha)$ with $N=10^{11}$ and $\alpha=2 /(e-1)$. We noted earlier that

$$
10^{11}=(10,32,17,6,8,15,11,9,0),
$$

and now observe that $Z_{i}$ is odd only for $i$ in $\{1,2,3,6\}$. Thus

$$
\llbracket i \text { even } \rrbracket \llbracket Z_{i} \text { odd } \rrbracket=\llbracket i \in\{2,6\} \rrbracket .
$$

We apply Theorem 4.4 to get

$$
\begin{aligned}
S_{100000000000}\left(\frac{2}{e-1}\right) & =-\sum_{i=0}^{8}\left(\prod_{t=i+1}^{8}(-1)^{Z_{t}}\right) \llbracket i \text { even } \rrbracket \llbracket Z_{i} \text { odd } \rrbracket \\
& =-\left(\prod_{t=2+1}^{8}(-1)^{Z_{t}}+\prod_{t=6+1}^{8}(-1)^{Z_{t}}\right) \\
& =-\left((-1)^{15+8+6+17+32+10}+(-1)^{32+10}\right)=-2 .
\end{aligned}
$$


A 1 Ghz PC running Mathematica would take about twenty days to compute this naively.

Proof of Theorem 4.4. Since $\alpha$ is fixed throughout this proof, and to avoid subscripts with subscripts, we simplify $S_{N}(\alpha)$ to $S(N)$. By definition $p_{0}=a_{0}, p_{1}=a_{0} a_{1}+1$, and $p_{i}=a_{i} p_{i-1}+p_{i-2}$, ensuring that all $p_{i}$ are odd. Idea 3 tells us that

$S\left(q_{i}\right)=S\left(q_{i-1}\right)-S\left(q_{i}-q_{i-1}\right)=S\left(q_{i-1}\right)-\left(S\left(q_{i-1}\right)-S\left(q_{i}-2 q_{i-1}\right)\right)=S\left(q_{i}-2 q_{i-1}\right)$,

whence

$$
S\left(q_{i}\right)=S\left(q_{i}-2 q_{i-1}\right)=S\left(q_{i}-4 q_{i-1}\right)=\cdots=S\left(q_{i}-a_{i} q_{i-1}\right)=S\left(q_{i-2}\right)
$$

and so

$$
S\left(q_{i}\right)=S\left(q_{i-2}\right)=S\left(q_{i-4}\right)=\cdots= \begin{cases}S\left(q_{-1}\right) & \text { if } i \text { is odd } \\ S\left(q_{0}\right) & \text { if } i \text { is even. }\end{cases}
$$

Now $S\left(q_{-1}\right)=S(0)=0$ and

$$
S\left(q_{0}\right)=S(1)=(-1)^{\lfloor\alpha\rfloor}=(-1)^{a_{0}}=-1,
$$

which completely solves the problem when $N$ is a denominator of a convergent to $\alpha$. Note that this gives

$$
S\left(q_{i}\right)=-\llbracket i \text { even } \rrbracket,
$$

which matches with the formula stated in the theorem. This will serve both as a basis for induction, and as a key step in the induction itself.

Suppose that the formula has been proved for all arguments less than $N=$ $\left(Z_{I}, \ldots, Z_{0}\right)$, with $I$ chosen so that $Z_{I} \neq 0$. We have two cases: either $Z_{I}>1$ or $Z_{I}=1$.

If $Z_{I}>1$ (the easy case, so we do it first), then applying Idea 3 twice yields (writing $q$ in place of $q_{I}$ )

$$
S(N)=S(q)-S(N-q)=S(q)-(S(q)-S(N-2 q))=S(N-2 q) .
$$

Since the formula we are proving cares only about the parity of $Z_{i}$, we need only to apply the induction hypothesis to $N-2 q_{I}=\left(Z_{I}-2, Z_{I-1}, \ldots, Z_{0}\right)$ to complete the proof.

If $Z_{I}=1$, then we have (again with $q=q_{I}$ )

$$
\begin{aligned}
S(N) & =S(q)-S\left(\left(Z_{I-1}, \ldots, Z_{0}\right)\right) \\
& =S(q)+\sum_{i=0}^{I-1}\left(\prod_{t=i+1}^{I-1}(-1)^{Z_{t}}\right) \llbracket i \text { even } \rrbracket \llbracket Z_{i} \text { odd } \rrbracket .
\end{aligned}
$$

We remark that we have used the induction hypothesis applied to $\left(Z_{I-1}, \ldots, Z_{0}\right)$, along with the fact that the formula is not affected if we pad the left of the CFE with zeros (since it may happen that $Z_{I-1}=0$, for example). We can expand the 
$S\left(q_{I}\right)$ term by observing that $S(q)=-\llbracket I$ even $\rrbracket$ by our base case, $\llbracket Z_{I}$ odd $\rrbracket=1$ since $Z_{I}=1$, and $\prod_{t=I+1}^{I}(-1)^{Z_{t}}=1$ because it is an empty product. Thus,

$$
S(q)=-\left(\prod_{t=I+1}^{I}(-1)^{Z_{t}}\right) \llbracket I \text { even } \rrbracket \llbracket Z_{I} \text { odd } \rrbracket .
$$

Further, since $Z_{I}=1, \prod_{t=i+1}^{I-1}(-1)^{Z_{t}}=-\prod_{t=i+1}^{I}(-1)^{Z_{t}}$ when $0 \leq i<I$, so

$$
\sum_{i=0}^{I-1}\left(\prod_{t=i+1}^{I-1}(-1)^{Z_{t}}\right) \llbracket i \text { even } \rrbracket \llbracket Z_{i} \text { odd } \rrbracket=-\sum_{i=0}^{I-1}\left(\prod_{t=i+1}^{I}(-1)^{Z_{t}}\right) \llbracket i \text { even } \rrbracket \llbracket Z_{i} \text { odd } \rrbracket .
$$

Equation (6) now reduces to the formula as stated in the theorem.

The record-holders. Recall that the record-holder at $k$ is defined by

$$
R_{k}(\alpha)=\min \left\{N \geq 0: S_{N}(\alpha)=k\right\} .
$$

Obviously $R_{0}(\alpha)=0$. The following corollary considers nonzero $k$ and $\alpha$ satisfying the hypotheses of Theorem 4.4

Corollary 4.5. Let $\alpha=\left[a_{0} ; a_{1}, a_{2}, \ldots\right]$, with $a_{0}$ odd and all other $a_{i}$ even. If $q_{i}$ are its continuants, then

$$
R_{k}(\alpha)=q_{2 k-1} \llbracket k>0 \rrbracket+\sum_{i=0}^{2|k|-2} q_{i} .
$$

Proof. We briefly discuss the case in which $k<0$; the "positive $k$ " case is similar and left to our most diligent readers. Consider

$$
N_{k}:=\sum_{i=0}^{2|k|-2} q_{i}
$$

It is not obvious that the CFE of $N_{k}$ is $(1,1, \ldots, 1)$. To verify this we must show that $\sum_{i=0}^{j} q_{i}<q_{j+1}$ for each $j$, which requires use of the hypothesis that $a_{i} \geq 2$ $(i>0)$.

Assuming this, we have $Z_{i}=1$ when $0 \leq i \leq 2|k|-2$, and

$$
\prod_{t=i+1}^{2|k|-2}(-1)^{Z_{t}} \llbracket i \text { even } \rrbracket=(-1)^{2|k|-2-i} \llbracket i \text { even } \rrbracket=\llbracket i \text { even } \rrbracket .
$$

In light of this, the conclusion of Theorem 4.4 reduces to the statement that

$$
S_{N}(\alpha)=-\sum_{i=0}^{2|k|-2} \llbracket i \text { even } \rrbracket=k,
$$

so $R_{k}(\alpha) \leq N_{k}$. 
Now suppose that $R_{k}(\alpha)=M=\left(Z_{I^{\prime}}, Z_{I^{\prime}-1}, \ldots, Z_{0}\right) \leq N_{k}$, where $I^{\prime} \leq 2|k|-2$ (since $M \leq N_{k}$ ). By taking the absolute value of each side of the formula given in Theorem 4.4 and invoking the inequality $\llbracket Z_{i}$ odd $\rrbracket \leq 1$, we find that

$$
|k|=\left|S_{M}(\alpha)\right| \leq \sum_{i=0}^{I^{\prime}} \llbracket i \text { even } \rrbracket=\left\lfloor\frac{I^{\prime}}{2}\right\rfloor+1 \leq \frac{I^{\prime}}{2}+1,
$$

and consequently that $I^{\prime} \geq 2|k|-2$. Thus, $I^{\prime}=2|k|-2$, and Theorem 4.4 now gives

$$
\begin{aligned}
k & =-\sum_{i=0}^{2|k|-2}\left(\prod_{t=i+1}^{2|k|-2}(-1)^{Z_{t}}\right) \llbracket i \text { even } \rrbracket \llbracket Z_{i} \text { odd } \rrbracket \\
& =-\sum_{s=0}^{|k|-1}\left(\prod_{t=2 s+1}^{2|k|-2}(-1)^{Z_{t}}\right) \llbracket Z_{2 s} \text { odd } \rrbracket .
\end{aligned}
$$

The sum over $s$ has $|k|$ terms, each with absolute value 0 or 1 , and the sum is $k$. It follows that,

$$
1=\llbracket Z_{2 s} \text { odd } \rrbracket \prod_{i=2 s+1}^{2|k|-2}(-1)^{Z_{i}}
$$

when $0 \leq s \leq-k-1$. For $s=|k|-1$ this implies that $Z_{2|k|-2}$ and $Z_{2|k|-1}$ are both odd, and taking successively smaller values for $s$ informs us that all $Z_{i}$ are odd $(0 \leq i \leq 2|k|-2)$. Thus $M \geq \sum_{i=0}^{2|k|-2} q_{i}=N_{k}$.

If $\alpha=\sqrt{m^{2}+1}-m+1$, as in Theorem 3.1. then we have explicit formulas for $q_{i}$ and $R_{k}(\alpha)$. It is simply a matter of arithmetic to turn these formulas into bounds on $\left|S_{N}\right|$ of the type given in Theorem 3.1

The next corollary, our finale, is in pleasant contrast to Theorem 3.1 .

Corollary 4.6. The value of $R_{k}(\sqrt{2})$ is given by

$$
R_{k}(\sqrt{2})= \begin{cases}\left\lfloor\frac{1}{4}(1+\sqrt{2})^{2 k+1}\right\rfloor & \text { if } k>0, \\ \left\lfloor\frac{1}{4}(1+\sqrt{2})^{-2 k}\right\rfloor & \text { if } k<0 .\end{cases}
$$

In particular, there are infinitely many $N$ with

$$
\left|S_{N}(\sqrt{2})\right|>\frac{\log (4 N)}{2 \log (1+\sqrt{2})}>\frac{\log N}{2 \log (1+\sqrt{2})}+0.78 .
$$

Proof. The continuants are given by $q_{0}=1, q_{1}=2$, and $q_{i}=2 q_{i-1}+q_{i-2}$. Solving this recurrence leads to

$$
q_{i}=\frac{(1+\sqrt{2})^{i+1}-(1-\sqrt{2})^{i+1}}{2 \sqrt{2}} .
$$


If $k<0$, we conclude that

$$
R_{k}(\sqrt{2})=\sum_{i=0}^{2|k|-2} q_{i}=-\frac{1}{2}+\frac{(1+\sqrt{2})^{-2 k}}{4}+\frac{(1-\sqrt{2})^{-2 k}}{4}=\left\lfloor\frac{(1+\sqrt{2})^{-2 k}}{4}\right\rfloor .
$$

We leave the positive $k$ case to the reader.

Now take $k<0$, and set $N=R_{k}(\sqrt{2})<(1+\sqrt{2})^{2|k|} / 4$. Solving for $|k|$, we find that

$$
\left|S_{N}(\sqrt{2})\right|=|k|>\frac{\log (4 N)}{2 \log (1+\sqrt{2})} .
$$

5. FOUR HARDER QUESTIONS. We now state four problems that we have been unable to resolve.

- What happens for other $\alpha$ ? For example, we know from section 2 that if $\alpha$ is any quadratic irrational, then $S_{N}(\alpha)=\mathcal{O}(\log N)$. Is this the correct type of growth for all quadratic irrationals? What are the necessary and sufficient conditions on $\alpha$ for $S_{N}(\alpha)=\mathcal{O}(\log N)$ in terms of the continued fraction expansion of $\alpha$ ? It seems unlikely that $S_{N}(\alpha)$ is $\mathcal{O}(\log N)$ for almost all $\alpha$, but a proof of this is elusive.

- What can be said for a given base $m$ about the number of $\lfloor n \alpha\rfloor(1 \leq n \leq N)$ that belong to the various congruence classes modulo $m$ ? There are $m-1$ free parameters here, because the sum is always $N$. For $m=2$, this is a problem equivalent to the one that we have worked on in this article; for $m \geq 3$ it is different. As a starting point, one may wish to consider the sum

$$
\sum_{n=1}^{N}\left(e^{2 \pi i / m}\right)^{\lfloor n \alpha\rfloor}
$$

- Our ideas do not generalize in any really straightforward manner to handle nonhomogeneous sequences. For example, is

$$
\left|\sum_{n=1}^{N}(-1)^{\lfloor n \sqrt{2}+1 / 2\rfloor}\right| \leq \frac{\log N}{2 \log (1+\sqrt{2})}+\frac{3}{2}
$$

when $N>2$ ?

- The problem of bounding $S_{N}(\sqrt{2})$ originally arose [19] in studying the convergence of

$$
\sum_{n=1}^{\infty} \frac{(-1)^{\lfloor n \sqrt{2}\rfloor}}{n}
$$

Is it possible to evaluate this infinite sum? Is it rational? Schmuland [21] studies the distribution of $\sum_{n=1}^{\infty} w_{n} / n$, where the $w_{n}$ are independent, identically 
distributed random variables taking the values 1 and -1 with equal probability. Does the $\operatorname{sum} \sum_{n=1}^{\infty}(-1)^{\lfloor n / \alpha\rfloor} / n$, where $\alpha$ is chosen uniformly from $(0,1)$, have the same distribution?

ACKNOWLEDGMENT. The first author thanks the National Science Foundation (grant DMS-0202460) for its support. The authors thank Dennis Eichhorn for carefully proofreading the manuscript.

\section{REFERENCES}

[1] S. Beatty, Problem 3173, Amer. Math. Monthly 33 (March 1926), 159, There was an error in the numbering of problems in 1926, and there are two 'Problem 3173's. S. Beatty's problem is in issue 3 (p. 159) and the other (unrelated) problem is in issue 4 (p. 228). Two solutions were published as solutions to Problem 3177 in vol. 34 (3), March 1927, pp 159-160. The first solution is given jointly by Ostrowski and Hyslop; the second solution is by Aitken.

[2] H. Behnke, Über die Verteilung von Irrationalitäten mod 1, Abh. Math. Semin. Univ. Hamburg 1 (1922), 252-267.

[3] _ Zur Theorie der diophantischen Approximationen I., Abh. Math. Semin. Univ. Hamburg 3 (1924), 261-318.

[4] D. Borwein and W. Gawronski, On certain sequences of plus and minus ones, Canad. J. Math. 30 (1978), 170-179.

[5] T. C. Brown, Descriptions of the characteristic sequence of an irrational, Canad. Math. Bull. 36 (1993), 15-21.

[6] H.-G. Carstens, W. A. Deuber, W. Thumser, and E. Koppenrade, Geometrical bijections in discrete lattices, Combin. Probab. Comput. 8 (1999), 109-129.

[7] M. Drmota and R. F. Tichy, Sequences, Discrepancies and Applications, Lecture Notes in Mathematics, Springer-Verlag, Berlin, 1997, ISBN 3-540-62606-9.

[8] A. S. Fraenkel, Systems of numeration, Amer. Math. Monthly 92 (1985), 105-114.

[9] R. K. Guy, Unsolved Problems in Number Theory, 2nd ed., Springer-Verlag, New York, 1994, ISBN 0-387-94289-0.

[10] G. H. Hardy and E. M. Wright, An Introduction to the Theory of Numbers, 5th ed., Clarendon Press, Oxford University Press, New York, 1979, ISBN 0-19-853170-2, 0-19-853171-0.

[11] M. Hata, Irrationality measures for $\pi$ and other mysterious numbers, Transcendental Number Theory and Related Topics, Kyungnam Univ., Masan, 1998, pp. 21-28.

[12] E. Hlawka, The Theory of Uniform Distribution, A B Academic Publishers, Berkhamsted, 1984, ISBN 0-907360-02-5.

[13] A. Khintchine, Ein Satz über Kettenbrüche mit arithmetischen Anwendungen, Math. Z. 18 (1923), 289-306.

[14] __ Einige Satze über Kettenbrüche, mit Anwendungen auf die Theorie der Diophantischen Approximationen, Math. Ann. 92 (1924), 115-125.

[15] D. E. Knuth, Two notes on notation, Amer. Math. Monthly 99 (1992), 403-422.

[16] I. Niven, H. S. Zuckerman, and H. L. Montgomery, An Introduction to the Theory of Numbers, 5th ed., John Wiley \& Sons, New York, 1991, ISBN 0-471-62546-9.

[17] K. O'Bryant, Fraenkel's theorem and Brown's decomposition, Integers 3 (2003), paper A11, 17 pp. (electronic).

[18] K. H. Rosen, Elementary Number Theory and Its Applications, 2nd ed., Addison-Wesley, Reading, MA, 1988, ISBN 0-201-11958-7.

[19] H. D. Ruderman, Problem 6105, Amer. Math. Monthly 83 (1976), 573. 
[20] W. M. Schmidt, Irregularities of distribution. VII, Acta Arith. 21 (1972), 45-50.

[21] B. Schmuland, Random harmonic series, Amer. Math. Monthly 110 (2003), 407-416.

[22] J. Schoißengeier, On the discrepancy of (no), Acta Arith. 44 (1984), 241-279.

[23] M. Serbinowska, A case of an almost alternating series, preprint.

[24] E. W. Weisstein, Random walk-1 dimensional (2003), Available at mathworld.wolfram.com

KEVIN O'BRYANT is a University of Illinois at Urbana-Champaign alumnus, and after a stint as an NSF postdoc (DMS 0202460) at the University of California, San Diego, he has taken a permanent position at the City University of New York. His research is mostly in combinatorial number theory and is strongly motivated by the philosophy of experimental mathematics. His hobbies include backgammon, nonstandard analysis, and drinking coffee. Department of Mathematics, City University of New York, CSI, Staten Island, NY 10314 kevin@member.ams.org

BRUCE REZNICK got his degrees from Caltech (B.S., 1973) and Stanford (Ph.D., 1976), and his been at the University of Illinois at Urbana-Champaign since 1979. He is interested in combinatorial problems in number theory, algebra, analysis, geometry, often involving polynomials. He still roots for the Chicago Cubs, but has forgotten why.

Department of Mathematics, University of Illinois at Urbana-Champaign, 1409 W. Green St., Urbana, IL 61801

reznick@math.uiuc.edu

MONIKA SERBINOWSKA enjoyed growing up in Warsaw, Poland. She obtained her M.S. from Warsaw University in 1990 under the supervision of Wojciech Guziǹski. In 1996 she obtained a Ph.D. from the University of Utah for work in theoretical statistics under the direction of Lajos Horváth. Currently, she is an assistant professor at Weber State University. She is now pursuing questions from number theory, continued fractions and geometry. She has three sons and she is an avid bridge player, hiker and skier.

Department of Mathematics, Weber State University, Ogden, UT 84408

mserbinowska@weber.edu 